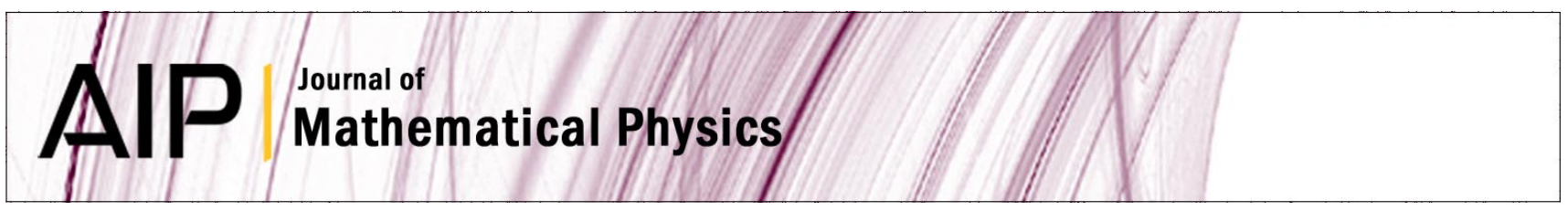

\title{
Integrable boundary value problems for elliptic type Toda lattice in a disk
}

Metin Gürses, Ismagil Habibullin, and Kostyantyn Zheltukhin

Citation: J. Math. Phys. 48, 102702 (2007); doi: 10.1063/1.2799256

View online: http://dx.doi.org/10.1063/1.2799256

View Table of Contents: http://jmp.aip.org/resource/1/JMAPAQ/v48/i10

Published by the American Institute of Physics.

\section{Additional information on J. Math. Phys.}

Journal Homepage: http://jmp.aip.org/

Journal Information: http://jmp.aip.org/about/about_the_journal

Top downloads: http://jmp.aip.org/features/most_downloaded

Information for Authors: http://jmp.aip.org/authors

\section{ADVERTISEMENT}

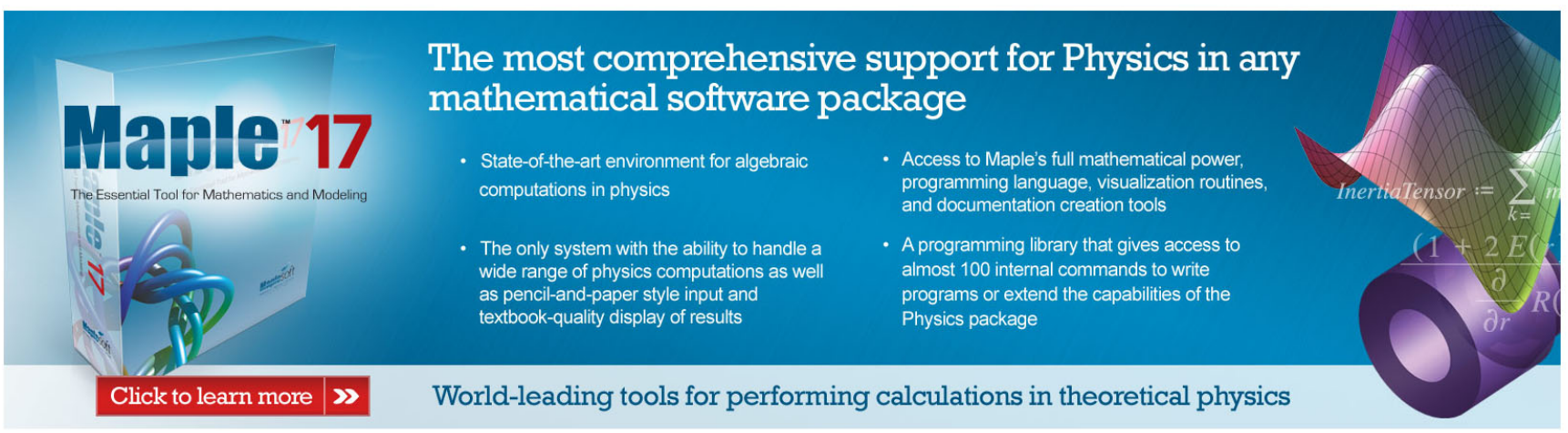




\title{
Integrable boundary value problems for elliptic type Toda lattice in a disk
}

\author{
Metin Gürses ${ }^{\text {a) }}$ and Ismagil Habibullin ${ }^{\text {b) }}$ \\ Department of Mathematics, Faculty of Sciences, Bilkent University, 06800 Ankara, \\ Turkey \\ Kostyantyn Zheltukhin ${ }^{\text {) }}$ \\ Department of Mathematics, Faculty of Sciences, Middle East Technical University, \\ 06531 Ankara, Turkey
}

(Received 16 June 2007; accepted 25 September 2007; published online 25 October 2007)

\begin{abstract}
The concept of integrable boundary value problems for soliton equations on $R$ and $R_{+}$is extended to regions enclosed by smooth curves. Classes of integrable boundary conditions in a disk for the Toda lattice and its reductions are found. (C) 2007 American Institute of Physics. [DOI: 10.1063/1.2799256]
\end{abstract}

\section{INTRODUCTION}

The inverse scattering transform method (ISM) discovered in 1967 has proved to be a powerful tool to construct exact solutions and to solve the Cauchy problem for a large variety of nonlinear integrable models of mathematical physics. However, real physical applications are usually related to mathematical models with boundary conditions. For this reason, the problem of adopting the ISM to a boundary value problem as well as to an initial boundary value (mixed) problem is very important. During the last two decades, this field of research has been intensively studied. It becomes clear that only special kinds of boundary conditions preserve the integrability property of the equation given. Different approaches were worked out to look for such classes of boundary conditions based on Hamiltonian structures, ${ }^{1}$ on higher symmetries, ${ }^{2-4}$ and the Lax representation. ${ }^{5,6}$ Integrable initial boundary value problems on a half-line (in $1+1$ case) or a half-plane (in $1+2$ case) for soliton equations nowadays is a rather studied subject. Analytical aspects have been developed in Refs. 7-11 where large classes of solutions were constructed. However, boundary value problem for the elliptic soliton equations or initial boundary value problem for regions with more complicated boundary is still much less investigated (see, Refs. 12-14).

If the boundary conditions are not consistent with the integrability property of the equation, then the standard version of the inverse scattering transform method cannot be applied to the corresponding boundary value problem. The method requires a very essential modification. Various ideas to extend the ISM to the initial boundary value problems are suggested in Refs. 12, $15-17$ and 20,21 .

In Refs. 5 and 6, an effective tool to search integrable boundary conditions has been proposed based on some special involutions of the auxiliary linear problem. This method (below for the sake of convenience, we refer it as the method of involutions) can be applied to integrable equations in both $(1+1)$ - and $(1+2)$-dimensional cases. Some examples of application of the inverse scattering transform method for such kind of boundary value problems were considered in Ref. 18.

\footnotetext{
${ }^{a)}$ Electronic mail: gurses@fen.bilkent.edu.tr

b) On leave from Ufa Institute of Mathematics, Russian Academy of Science, Chernyshevskii Str., 112, Ufa 450077, Russia. Electronic mail: habibullin_i@mail.rb.ru and habib@fen.bilkent.edu.tr

${ }^{c)}$ Electronic mail: zheltukh@metu.edu.tr
} 
In this article, we show that the method of involutions allows one to extend the concept of integrability to boundary value problems on bounded (unbounded) regions enclosed by any closed smooth curve.

Let us explain briefly the approach we use. We call boundary value problem integrable if it admits a Lax pair. Because of this reason, we look for a boundary condition simultaneously with its Lax representation. The starting point is to make a correct assumption about the possible form of the Lax pair of the boundary value problem. Actually, this Lax pair is made up from several different Lax pairs of the original equation itself by gluing the eigenfunctions along the boundary by properly chosen additional boundary conditions. As examples, we take the Liouville equation and the two-dimensional Toda lattice equation. To generate new Lax pairs, we use point symmetries (involutions) which leave invariant the nonlinear equation under consideration but change its Lax pair.

In Sec. II, as a trial example, we consider the Liouville equation. We remind the definition of integrable boundary conditions and find an example of integrable boundary conditions on a circle with its Lax representation (see the list at the end of the second section).

In Sec. III, we study the two-dimensional Toda lattice equation on a circular cylinder: $r$ $<a, 0 \leqslant \theta \leqslant 2 \pi,-\infty<n<\infty$. Several types of integrable boundary value problems for this lattice and Lax representations of the boundary value problems are found by using the method of involutions (see the list at the end of the third section).

In Sec. IV, we consider periodicity closure constraints reducing the Toda lattice to the sinhGordon and Tzitzeica equations.

In Sec. V, we give a class of exact solutions of the Toda lattice on a circle with a nonhomogeneous Neumann-type boundary condition in a disk.

\section{LIOUVILLE EQUATION}

In this section, we concentrate on boundary value problems for elliptic equations. Suppose that the boundary $\Gamma$ of a domain $D$ is parametrized by the equation $x^{\prime}=f\left(t^{\prime}\right)$ that introduces a local system of coordinates by taking the $t$ axis along the tangent direction and the $x$ axis along the normal direction to the curve $\Gamma$.

Suppose that the differential equation under consideration,

$$
E(u)=0,
$$

admits two different Lax representations. For the sake of simplicity, we take them rewritten in terms of the new coordinates

$$
\begin{gathered}
Y_{x}=U\left(\lambda, u, u_{x}, \ldots\right) Y(\lambda), \\
Y_{t}=V\left(\lambda, u, u_{x}, \ldots\right) Y(\lambda)
\end{gathered}
$$

and

$$
\begin{aligned}
& \tilde{Y}_{x}=\tilde{U}\left(\tilde{\lambda}, u, u_{x}, \ldots\right) \tilde{Y}(\tilde{\lambda}), \\
& \tilde{Y}_{t}=\tilde{V}\left(\tilde{\lambda}, u, u_{x}, \ldots\right) \tilde{Y}(\tilde{\lambda}),
\end{aligned}
$$

where $\lambda, \tilde{\lambda}$ are spectral parameters. Now, the equation of the boundary is of the form $x=0$. We are looking for conditions that allow to relate the equations for $t$ evolution along the boundary since $x$ is fixed. More precisely, we have the following definition.

Definition 1: A boundary condition

$$
\Omega\left(t, u, u_{t}, u_{x}, \ldots\right)=0
$$

is integrable if there exists a matrix $F(\lambda, t, u, \ldots)$ and function $h(\lambda)$ such that on the boundary 
$x=0$, the function $Y=F(\lambda, t, u, \ldots) \tilde{Y}(\tilde{\lambda})$ is a solution of the equation $Y_{t}=V Y$ for any solution $\tilde{Y}$ of the equation $\tilde{Y}_{t}=\tilde{V} \tilde{Y}$ with $\tilde{\lambda}=h(\lambda)$, provided that the boundary condition holds.

If a boundary condition is integrable in the sense of the definition above, this means that the corresponding boundary value problem admits the Lax representation consisting of the two Lax pairs (2) and (3) defined on the domain $D$ such that the eigenfunctions $Y$ and $\tilde{Y}$ satisfy along the boundary an additional boundary condition $\left.(Y-F \tilde{Y})\right|_{\Gamma}=0$.

To consider a circle as a boundary, we use polar coordinates $(r, \theta)$. So, the boundary is $r=a$. In polar coordinates, the Liouville equation is

$$
u_{r r}+\frac{1}{r} u_{r}+\frac{1}{r^{2}} u_{\theta \theta}=8 e^{u}
$$

It admits the Lax pair

$$
Y_{r}=L Y, \quad Y_{\theta}=A Y,
$$

where $x=r, t=\theta, U=L, V=A$, and

$$
\begin{gathered}
L=\left(\begin{array}{cc}
\frac{e^{u+i \theta}}{2 \lambda}+\lambda e^{-i \theta} & -\frac{e^{u+i \theta}}{2 \lambda}+\frac{1}{4} u_{r}+\frac{i}{4 r} u_{\theta} \\
\frac{e^{u+i \theta}}{2 \lambda}+\frac{1}{4} u_{r}+\frac{i}{4 r} u_{\theta} & -\frac{e^{u+i \theta}}{2 \lambda}-\lambda e^{-i \theta}
\end{array}\right), \\
A=i r\left(\begin{array}{cc}
\frac{e^{u+i \theta}}{2 \lambda}-\lambda e^{-i \theta} & -\frac{e^{u+i \theta}}{2 \lambda}-\frac{1}{4} u_{r}-\frac{i}{4 r} u_{\theta} \\
\frac{e^{u+i \theta}}{2 \lambda}-\frac{1}{4} u_{r}-\frac{i}{4 r} u_{\theta} & -\frac{e^{u+i \theta}}{2 \lambda}+\lambda e^{-i \theta}
\end{array}\right) .
\end{gathered}
$$

To obtain a second Lax representation, we use the Kelvin transformation.

Equation (5) is invariant under the Kelvin transformation

$$
\bar{r}=\frac{a^{2}}{r}, \quad \bar{u}=u+4 \ln \frac{a}{r} .
$$

Under such transformation, the Lax pair (6) takes the form

$$
\bar{Y}_{r}=\bar{L} \bar{Y}, \quad \bar{Y}_{\theta}=\bar{A} \bar{Y},
$$

where

$$
\bar{L}=\left(\begin{array}{cc}
\frac{r^{4} e^{u+i \theta}}{2 a^{4} \tilde{\lambda}}+\tilde{\lambda} e^{-i \theta} & -\frac{r^{4} e^{u+i \theta}}{2 a^{4} \lambda}-\frac{r^{2} u_{r}}{4 a^{2}}-\frac{r}{a^{2}}+\frac{i r}{4 a^{2}} u_{\theta} \\
\frac{r^{4} e^{u+i \theta}}{2 a^{2} \tilde{\lambda}}-\frac{r^{2} u_{r}}{4 a^{2}}-\frac{r}{a^{2}}+\frac{i r}{4 a^{2}} u_{\theta} & -\frac{r^{4} e^{u+i \theta}}{2 a^{4} \tilde{\lambda}}-\tilde{\lambda} e^{-i \theta}
\end{array}\right),
$$




$$
\bar{A}=\frac{i a^{2}}{r}\left(\begin{array}{cc}
\frac{r^{4} e^{u+i \theta}}{2 a^{4} \tilde{\lambda}}-\tilde{\lambda} e^{-i \theta} & -\frac{r^{4} e^{u+i \theta}}{2 a^{4} \tilde{\lambda}}+\frac{r^{2} u_{r}}{4 a^{2}}+\frac{r}{a^{2}}-\frac{i r u_{\theta}}{4 a^{2}} \\
\frac{r^{4} e^{u+i \theta}}{2 a^{4} \tilde{\lambda}}+\frac{r^{2} u_{r}}{4 a^{2}}+\frac{r}{a^{2}}-\frac{i r u_{\theta}}{4 a^{2}} & -\frac{r^{4} e^{u+i \theta}}{2 a^{4} \tilde{\lambda}}+\tilde{\lambda} e^{-i \theta}
\end{array}\right) .
$$

We will look for a boundary condition under which there exists a transformation $\tilde{\lambda}=h(\lambda)$ and a nondegenerate matrix $F(\lambda, \theta, u)$ such that $Y(\lambda)=F \tilde{Y}(h(\lambda))$ will solve the $Y_{\theta}(\lambda)=\left.A\right|_{r=a} Y(\lambda)$ for every solution $\tilde{Y}(\tilde{\lambda})$ of the equation $\tilde{Y}_{\theta}(\tilde{\lambda})=\left.\widetilde{A}\right|_{r=a} \tilde{Y}(\tilde{\lambda})$.

Lemma 1: The integrable boundary condition is given by

$$
\left.u_{r}\right|_{r=a}=\frac{-2}{a},
$$

and there are two choices for the matrix $F$ and the function $h$,

(i)

$$
h(\lambda)=\lambda, \quad F=\left(\begin{array}{ll}
1 & 0 \\
0 & 1
\end{array}\right),
$$

(ii)

$$
h(\lambda)=-\lambda, \quad F=\left(\begin{array}{cc}
0 & -1 \\
-1 & 0
\end{array}\right) .
$$

Proof: Let $\bar{Y}(\lambda)$ satisfy the equation $\bar{Y}_{\theta}=\bar{A} \bar{Y}$. On the boundary $r=a, Y=F \bar{Y}(h(\lambda))$ has to satisfy $Y_{\theta}=A Y$. Substituting $Y=F \bar{Y}(h(\lambda))$ into $Y_{\theta}=A Y$ and using $\bar{Y}_{\theta}=\bar{A} \bar{Y}$ for $\bar{Y}_{\theta}(h(\lambda))$, we obtain

$$
\left(\frac{\mathrm{d}}{\mathrm{d} \theta} F-A(\lambda) F+F \bar{A}(h(\lambda))\right) \bar{Y}(h(\lambda))=0 .
$$

The above equality holds if

$$
\frac{\mathrm{d}}{\mathrm{d} \theta} F=A(\lambda) F-F \bar{A}(h(\lambda)) .
$$

We have an equation for the unknown matrix $F$ and function $h(\lambda)$. To solve the boundary condition (4) with respect to $u_{r}$, we let $u_{r}=G\left(\theta, u, u_{\theta}\right)$. Assuming that $F$ does not depend on $u_{\theta}$ and differentiating (17) twice with respect to $u_{\theta}$, we obtain $\partial^{2} u_{r} / \partial u_{\theta}^{2}=0$. That is, $u_{r}=g_{1}(u, \theta) u_{\theta}$ $+g_{2}(u, \theta)$. We substitute the above expression for $u_{r}$ into (17) and let

$$
F=\left(\begin{array}{ll}
f_{11}(u, \lambda, \theta) & f_{12}(u, \lambda, \theta) \\
f_{21}(u, \lambda, \theta) & f_{22}(u, \lambda, \theta)
\end{array}\right) .
$$

Separating terms with $u_{\theta}$ and without $u_{\theta}$ in (17), we obtain two sets of equations. We write the first set of equations, terms with $u_{\theta}$, as

$$
\frac{\partial}{\partial u} f=P f,
$$

where $f=\left(f_{11}, f_{12}, f_{21}, f_{22}\right)^{T}$ and $P$ is a matrix 


$$
i a\left(\begin{array}{cccc}
0 & -\left(\frac{g_{1}}{4}-\frac{i}{4 a}\right) & \left(-\frac{g_{1}}{4}-\frac{i}{4 a}\right) & 0 \\
-\left(\frac{g_{1}}{4}-\frac{i}{4 a}\right) & 0 & 0 & \left(-\frac{g_{1}}{4}-\frac{i}{4 a}\right) \\
\left(-\frac{g_{1}}{4}-\frac{i}{4 a}\right) & 0 & 0 & -\left(\frac{g_{1}}{4}-\frac{i}{4 a}\right) \\
0 & \left(-\frac{g_{1}}{4}-\frac{i}{4 a}\right) & -\left(\frac{g_{1}}{4}-\frac{i}{4 a}\right) & 0
\end{array}\right) .
$$

We write the second set of equations, terms without $u_{\theta}$, as

$$
\frac{\partial}{\partial \theta} f=Q f
$$

where $Q$ is a matrix

$$
i a\left(\begin{array}{cccc}
\mu-\nu & -\left(\delta+\frac{e^{u+i \theta}}{2 h(\lambda)}\right) & \left(-\frac{g_{2}}{4}-\frac{e^{u+i \theta}}{2 \lambda}\right) & 0 \\
-\left(\delta-\frac{e^{u+i \theta}}{2 h(\lambda)}\right) & \mu+\nu & 0 & \left(-\frac{g_{2}}{4}-\frac{e^{u+i \theta}}{2 \lambda}\right) \\
\left(\frac{e^{u+i \theta}}{2 \lambda}-\frac{g_{2}}{4}\right) & 0 & -\mu-\nu & \left(\delta+\frac{e^{u+i \theta}}{2 h(\lambda)}\right) \\
0 & \left(\frac{e^{u+i \theta}}{2 \lambda}-\frac{g_{2}}{4}\right) & -\left(\delta-\frac{e^{u+i \theta}}{2 h(\lambda)}\right) & -\mu+\nu
\end{array}\right),
$$

with $\mu=e^{u+i \theta} / 2 \lambda-\lambda e^{-i \theta}, \nu=e^{u+i \theta} / 2 h(\lambda)-h(\lambda) e^{-i \theta}$, and $\delta=g_{2} / 4+1 / a$. tion:

Equations (19) and (21) must be compatible. This leads to the following compatibility condi-

$$
\left(P_{\theta}-Q_{u}+[P, Q]\right) f=0,
$$

where $[P, Q]$ is a commutator of $P$ and $Q$. The matrix $\left(P_{\theta}-Q_{u}+[P, Q]\right)$ is nonzero. To have a nonzero solution $f$, the determinant of $\left(P_{\theta}-Q_{u}+[P, Q]\right)$ must be zero. It gives the following equality:

$$
\frac{a^{6} e^{-4 i \theta}}{16}\left(h^{2}(\lambda)\left(a g_{1}(u, \theta)-i\right)^{2}-\left(a g_{1}(u, \theta)+i\right)^{2} \lambda^{2}\right)^{2}=0 .
$$

The above equality holds if either

(1) $h(\lambda)=\beta \lambda$ and $g_{1}=i(1+\beta) / a(1-\beta)$, where $\beta \in \mathbb{R} \backslash\{-1,1\}$ or

(2) $h(\lambda)=\lambda$ and $g_{1}=0$ or

(3) $h(\lambda)=-\lambda$ and $g_{1}=0$.

One can show that in case (1), there is no vector $f$ to satisfy Eqs. (19) and (21). In case (2), one has the only solution $f=q(\lambda)(1,0,0,1)^{T}$ if $g_{2}=-2 / a$. This gives the boundary condition (13), function $h$, and matrix $F$ given by (14).

Case (3) is similar to Case (2) and gives the same boundary condition (13), function $h$, and matrix $F$ given by (15).

From the above lemma, we have the following integrable boundary value problem with corresponding Lax pairs (we have two Lax pairs for the problem): 
(1)

$$
\begin{gathered}
r<a, \quad u_{r r}+\frac{1}{r} u_{r}+\frac{1}{r^{2}} u_{\theta \theta}=8 e^{u}, \\
Y_{r}(\lambda)=L(\lambda) Y(\lambda), \quad \bar{Y}_{r}(\lambda)=\bar{L}(\lambda) \bar{Y}(\lambda), \\
Y_{\theta}(\lambda)=A(\lambda) Y(\lambda), \quad \bar{Y}_{\theta}(\lambda)=\bar{A}(\lambda) \bar{Y}(\lambda), \\
r=a,\left.\quad u_{r}\right|_{r=a}=-\frac{2}{a}, \\
Y=F \bar{Y},
\end{gathered}
$$

where $F$ is given by (14), $L$ is given by (7), $A$ is given by (8), $\bar{L}$ is given by (11), and $\bar{A}$ is given by (12).

$$
\begin{gathered}
r<a, \quad u_{r r}+\frac{1}{r} u_{r}+\frac{1}{r^{2}} u_{\theta \theta}=8 e^{u}, \\
Y_{r}(\lambda)=L(\lambda) Y(\lambda), \quad \bar{Y}_{r}(-\lambda)=\bar{L}(-\lambda) \bar{Y}(-\lambda), \\
Y_{\theta}(\lambda)=A(\lambda) Y(\lambda), \quad \bar{Y}_{\theta}(-\lambda)=\bar{A}(-\lambda) \bar{Y}(-\lambda), \\
r=a,\left.\quad u_{r}\right|_{r=a}=-\frac{2}{a}, \\
Y(\lambda)=F \bar{Y}(-\lambda),
\end{gathered}
$$

where $F$ is given by (15), $L$ is given by (7), $A$ is given by (8), $\bar{L}$ is given by (11), and $\bar{A}$ is given by (12).

Remark 1: The above boundary value problem admits infinitely many explicit solutions of the form

$$
u=\ln \left(\frac{n^{2}\left(\alpha^{2}+\beta^{2}\right)}{4 r^{2}\left(\alpha(\cos n \theta+\beta \sin n \theta)^{2}\right.}\right),
$$

for any $\alpha, \beta, n$ and of the form

$$
u=-2 \ln \left(k\left(r^{2}+a^{2}\right)+r(\alpha \cos \theta+\beta \sin \theta)\right),
$$

where $\alpha^{2}+\beta^{2}=4+4 k^{2} a^{2}$. We note that all these solutions have a singularity inside the region $r$ $<a$. Unfortunately, we failed to find regular solutions to the above boundary value problem.

\section{TWO-DIMENSIONAL TODA LATTICE}

We make the same assumption, as in the case of the Liouville equation, for the coordinates. Hence, boundary is given by $x=0$. Again, we suppose that the differential equation under consideration admits two different Lax representations, 


$$
\begin{array}{ll}
Y_{x}=U Y, & \tilde{Y}_{x}=\tilde{U} \tilde{Y}, \\
Y_{t}=V Y, \quad \tilde{Y}_{t}=\tilde{V} \tilde{Y} .
\end{array}
$$

For the two-dimensional Toda lattice equation, $U, V, \widetilde{U}$, and $\widetilde{V}$ in (27) are linear operators.

Definition 2: A boundary condition

$$
\Omega(u)=0
$$

is integrable if there exists a linear differential operator $A$ such that on the boundary $x=0$, we have that $\widetilde{Y}=A Y$ is a solution of $\widetilde{Y}_{t}=\tilde{V} \widetilde{Y}$ for any solution $Y$ of $Y_{t}=V Y$, provided that the boundary condition holds.

To consider a circle as a boundary, we use polar coordinates $(r, \theta)$. So, the boundary is $r=a$. The two-dimensional Toda lattice equation in polar coordinates becomes

$$
u_{r r}+\frac{1}{r} u_{r}+\frac{1}{r^{2}} u_{\theta \theta}=\omega(n-1)-\omega(n),
$$

where $\omega(n)=\exp (u(n)-u(n+1))$. The above equation admits a Lax pair

$$
\begin{gathered}
\varphi_{1, r}(n)=\frac{e^{i \theta}}{2} \varphi_{1}(n+1)-\frac{1}{2}\left(u_{r}(n)-\frac{i}{r} u_{\theta}(n)\right) \varphi_{1}(n)-\frac{e^{-i \theta}}{2} \omega(n-1) \phi_{1}(n-1), \\
\varphi_{1, \theta}(n)=\frac{i r e^{i \theta}}{2} \varphi_{1}(n+1)-\frac{i r}{2}\left(u_{r}(n)-\frac{i}{r} u_{\theta}(n)\right) \varphi_{1}(n)+\frac{i r e^{-i \theta}}{2} \omega(n-1) \varphi_{1}(n-1) .
\end{gathered}
$$

To obtain other Lax representations, we use symmetries of Eq. (29).

(1) Reflection on $\theta$,

$$
\tilde{\theta}=-\theta \text {. }
$$

(2) The Kelvin transformation,

$$
\tilde{r}=\frac{a}{r}, \quad \tilde{u}=u+4 n \ln \frac{a}{r} .
$$

(3) Reflection on $n$,

$$
\widetilde{u}=-u(-n) .
$$

Using the transformation (32), we obtain the following Lax representation:

$$
\begin{gathered}
\varphi_{2, r}(n)=\frac{e^{-i \theta}}{2} \varphi_{2}(n+1)-\frac{1}{2}\left(u_{r}(n)+\frac{i}{r} u_{\theta}(n)\right) \varphi_{2}(n)-\frac{e^{i \theta}}{2} \omega(n-1) \varphi_{2}(n-1), \\
\varphi_{2, \theta}(n)=\frac{i r e^{-i \theta}}{2} \varphi_{2}(n+1)-\frac{i r}{2}\left(u_{r}(n)+\frac{i}{r} u_{\theta}(n)\right) \varphi_{2}(n)+\frac{i r e^{i \theta}}{2} \omega(n-1) \varphi_{2}(n-1) .
\end{gathered}
$$

Using the Kelvin transformation (33), we obtain the following Lax representation:

$$
\varphi_{3, r}(n)=\frac{e^{i \theta}}{2} \varphi_{3}(n+1)-\frac{1}{2}\left(\frac{-r^{2}}{a^{2}} u_{r}(n)+4 n \frac{r}{a^{2}}-\frac{i r}{a^{2}} u_{\theta}\right) \varphi_{4}(n)-\frac{r^{4} e^{-i \theta}}{2 a^{4}} \omega(n-1) \varphi_{3}(n-1),
$$




$$
\varphi_{3, \theta}(n)=\frac{i a^{2} e^{i \theta}}{2 r} \varphi_{3}(n+1)-\frac{i a^{2}}{2 r}\left(\frac{-r^{2}}{a^{2}} u_{r}(n)+4 n \frac{r}{a^{2}}-\frac{i r}{a^{2}} u_{\theta}\right) \varphi_{3}(n)+\frac{i r^{3} e^{-i \theta}}{2 a^{2}} \omega(n-1) \varphi_{3}(n-1)
$$

Using the transformations (34), we obtain the following Lax representation:

$$
\begin{gathered}
\varphi_{4, r}(n)=\frac{e^{i \theta}}{2} \varphi_{4}(n-1)-\frac{1}{2}\left(-u_{r}(n)+\frac{i}{r} u_{\theta}(n)\right) \varphi_{4}(n)-\frac{e^{-i \theta}}{2} \omega(n) \varphi_{4}(n+1), \\
\varphi_{4, \theta}(n)=\frac{i r e^{i \theta}}{2} \varphi_{4}(n-1)-\frac{i r}{2}\left(-u_{r}(n)+\frac{i}{r} u_{\theta}(n)\right) \varphi_{4}(n)+\frac{i r e^{-i \theta}}{2} \omega(n) \varphi_{4}(n+1) .
\end{gathered}
$$

According to Definition 2, to obtain the integrable boundary conditions, we relate the equations for $\theta$ evolution of the above Lax representations, on the boundary $r=a$. We consider the case when the eigenfunctions are related by the multiplication operator $\varphi_{i}=A(\theta, n, u, \ldots) \cdot \varphi_{j}$.

It turns out (see Lemma (7)) that Lax pairs corresponding to the Kelvin transformation (33) and the symmetry (34) are gauge equivalent. A solution of (38) transforms to a solution of (40) without any boundary conditions. So, some boundary value problems have two possible Lax pairs.

In Lemma 2, we derive the first boundary value problem in the list at the end of this section.

Lemma 2: Let $\varphi_{1}(n)$ be a solution of Eq. (31), then on the boundary $r=a$, a function $\varphi_{2}(n)$ $=A \cdot \varphi_{1}(n)$, where

$$
A=e^{2 i n+g(\theta)}, \quad g(\theta) \text { is an arbitrary function of } \theta,
$$

is a solution of Eq. (36), provided, that the following boundary condition:

$$
u(n)=2 \operatorname{in} \theta+g(\theta)+k(n), \quad k(n) \text { is an arbitrary function of } n,
$$

holds for all $n$.

Proof: On the boundary $r=a$, we substitute $\varphi_{2}(n)=A(n, \theta, u, \ldots) \cdot \varphi_{1}(n)$ into Eq. (36) and use (31) for $\varphi_{1, \theta}(n)$. The resulting equation holds if the coefficients of $\varphi_{1}(n+1), \varphi_{1}(n)$, and $\varphi_{1}(n$ $-1)$ are zero. Thus, we obtain

$$
\begin{gathered}
\frac{i a e^{i \theta}}{2} A(n)=\frac{i a e^{-i \theta}}{2} A(n+1), \\
A_{\theta}(n)-\frac{i a}{2}\left(u_{r}(n)-\frac{i u_{\theta}(n)}{a}\right) A(n)=-\frac{i a}{2}\left(u_{r}(n)+\frac{i u_{\theta}(n)}{a}\right) A(n), \\
\frac{i a e^{-i \theta}}{2} \omega(n-1) A(n)=\frac{i a e^{i \theta}}{2} \omega(n-1) A(n-1) .
\end{gathered}
$$

From Eqs. (43) and (45), we have that $A(n)=e^{2 i \theta} A(n-1)$. Hence, $A(n)=e^{2 i \theta n} b(\theta)$, where $b(\theta)$ is a function of $\theta$ only. Substituting $A(n)=e^{2 i \theta n} b(\theta)$ into Eq. (44), we obtain

$$
b_{\theta}+\left(2 i n-u_{\theta}(n)\right) b=0 .
$$

Since the function $b$ does not depend on $n$, we have that the coefficient of $b$ in the above equation does not depend on $n$, so $u_{\theta}(n)=2 i n+h(\theta)$. Integrating with respect to $\theta$, we obtain the boundary condition (42), where $k$ is an arbitrary function of $n$ and $g(\theta)=\int h(\theta) \mathrm{d} \theta$. Then, solving Eq. (44), assuming that the found boundary condition holds, we obtain $A=e^{2 i n+\int g(\theta) \mathrm{d} \theta}$, the expression (41) for $A$.

In a similar way, from the next lemmas, we derive other boundary value problems in the list. 
In Lemma 3 and Lemma 4, we derive the Lax pair for the second boundary value problem in the list.

Lemma 3: Let $\varphi_{1}(n)$ be a solution of Eq. (31), then on the boundary $r=a$, a function $\varphi_{3}(n)$ $=A \cdot \varphi_{1}(n)$, where

$$
A=e^{i a \int g(\theta) \mathrm{d} \theta}, \quad g(\theta) \text { is an arbitrary function of } \theta,
$$

is a solution of Eq. (38), provided that the following boundary condition:

$$
u_{r}(n)=\frac{2 n}{a}+g(\theta)
$$

holds for all $n$.

Proof: On the boundary $r=a$, we substitute $\varphi_{3}(n)=A(n) \cdot \varphi_{1}(n)$ into Eq. (38) and use (31) for $\varphi_{1, \theta}(n)$. The resulting equation holds if the coefficients of $\varphi_{1}(n+1), \varphi_{1}(n)$, and $\varphi_{1}(n-1)$ are zero. Thus, we obtain

$$
\begin{gathered}
\frac{i a e^{i \theta}}{2} A(n)=\frac{i a e^{i \theta}}{2} A(n+1), \\
A_{\theta}(n)-\frac{i a}{2}\left(u_{r}(n)-\frac{i u_{\theta}(n)}{a}\right) A(n)=\frac{i a}{2}\left(u_{r}(n)-\frac{4 n}{a}+\frac{i u_{\theta}(n)}{a}\right) A(n), \\
\frac{i a e^{-i \theta}}{2} \omega(n-1) A(n)=\frac{i a e^{-i \theta}}{2} \omega(n-1) A(n-1) .
\end{gathered}
$$

From Eqs. (49) and (51), we have that $A$ does not depend on $n$. Hence, the coefficient of $A$ in (50) must be a function of $\theta$ only. This gives the boundary condition (48). Then, solving Eq. (50), assuming that (48) holds, we obtain the expression (47) for $A$.

Lemma 4: Let $\varphi_{1}(n)$ be a solution of Eq. (31), then on the boundary $r=a$, a function $\varphi_{4}(n)$ $=A \cdot \varphi_{1}(n)$, where

$$
A=e^{2 i \theta n+u(n)+i a \int g(\theta) \mathrm{d} \theta}, g(\theta) \text { is an arbitrary function of } \theta,
$$

is a solution of Eq. (38), provided that the following boundary condition:

$$
u_{r}(n)=\frac{2 n}{a}+g(\theta)
$$

holds for all $n$.

Proof: On the boundary $r=a$, we substitute $\varphi_{4}(n)=A(n, \theta, u, \ldots) \cdot \varphi_{1}(n)$ into Eq. (40) and (38) and use (38) for $\varphi_{1, \theta}(n)$. The resulting equation holds if the coefficients of $\varphi_{2}(n+1), \varphi_{2}(n)$, and $\varphi_{2}(n-1)$ are zero. Thus, we obtain

$$
\begin{gathered}
\frac{i a e^{i \theta}}{2} A(n)=\frac{i a e^{-i \theta}}{2} \omega(n) A(n+1), \\
A_{\theta}(n)-\frac{i a}{2}\left(u_{r}(n)-\frac{i u_{\theta}(n)}{a}\right) A(n)=-\frac{i a}{2}\left(-u_{r}(n)+\frac{i u_{\theta}(n)}{a}\right) A(n),
\end{gathered}
$$




$$
\frac{i a e^{-i \theta}}{2} \omega(n-1) A(n)=\frac{i a e^{i \theta}}{2} A(n-1) .
$$

From Eqs. (54) and (56), we have that $A(n)=e^{-2 i \theta} \omega(n) A(n+1)$. Hence, $A(n)=e^{2 i \theta n+u(n)} b(\theta)$, where $F(\theta)$ is a function of $\theta$ only. Substituting $A(n)=e^{-2 i \theta n} b(\theta)$ into Eq. (55), we obtain

$$
b_{\theta}-\left(2 i n-i a u_{r}(n)\right) b=0 .
$$

Since the function $b$ does not depend on $n$, we have that the coefficient of $b$ in the above equation does not depend on $n$. This gives the boundary condition (53). Then, solving Eq. (55), assuming that the found boundary condition holds, we obtain the expression (52) for $A$.

In Lemma 5 and Lemma 6, we derive the Lax pair for the third boundary value problem in the list.

Lemma 5: Let $\varphi_{2}(n)$ be a solution of Eq. (36), then on the boundary $r=a$, a function $\varphi_{3}(n)$ $=A \cdot \varphi_{2}(n)$, where

$$
A=e^{-2 i \theta n+i a \int g(\theta) \mathrm{d} \theta}, \quad g(\theta) \text { is an arbitrary function of } \theta,
$$

is a solution of Eq. (38), provided that the following boundary condition:

$$
u_{r}(n)=-\frac{i}{a} u_{\theta}(n)+g(\theta),
$$

holds for all $n$.

Proof: On the boundary $r=a$, we substitute $\varphi_{3}(n)=A(n, \theta, u, \ldots) \cdot \varphi_{2}(n)$ into Eq. (38) and use (36) for $\varphi_{2, \theta}(n)$. The resulting equation holds if the coefficients of $\varphi_{2}(n+1), \varphi_{2}(n)$, and $\varphi_{2}(n$ -1) are zero. Thus, we obtain

$$
\begin{aligned}
\frac{i a e^{-i \theta}}{2} A(n) & =\frac{i a e^{i \theta}}{2} A(n+1), \\
A_{\theta}(n)-\frac{i a}{2}\left(u_{r}(n)+\frac{i u_{\theta}(n)}{a}\right) A(n) & =-\frac{i a}{2}\left(-u_{r}(n)+\frac{4 n}{r}-\frac{i u_{\theta}(n)}{a}\right) A(n), \\
\frac{i a e^{i \theta}}{2} A(n) & =\frac{i a e^{-i \theta}}{2} A(n-1) .
\end{aligned}
$$

From Eqs. (60) and (62), we have that $A(n)=e^{-2 i \theta} A(n-1)$. Hence, $A(n)=e^{-2 i \theta n} b(\theta)$, where $b(\theta)$ is a function of $\theta$ only. Substituting $A(n)=e^{-2 i \theta n} b(\theta)$ into Eq. (61), we obtain

$$
b_{\theta}-i a\left(u_{r}(n)+\frac{i}{a} u_{\theta}(n)\right) b=0 .
$$

Since the function $b$ does not depend on $n$, we have that the coefficient of $b$ in the above equation does not depend on $n$. This gives the boundary condition (59). Then, solving Eq. (61), assuming that the found boundary condition holds, we obtain the expression (58) for $A$.

Lemma 6: Let $\varphi_{2}(n)$ be a solution of Eq. (36), then on the boundary $r=a$, a function $\varphi_{4}(n)$ $=A \cdot \varphi_{2}(n)$, where

$$
A=e^{2 u(n)+\int g(\theta) \mathrm{d} \theta}, \quad g(\theta) \text { is an arbitrary function of } \theta,
$$

is a solution of Eq. (40), provided that the following boundary condition:

$$
u_{r}(n)=-\frac{i}{a} u_{\theta}(n)+g(\theta),
$$


holds for all $n$.

Proof: On the boundary $r=a$, we substitute $\varphi_{4}(n)=A(n, \theta, u, \ldots) \cdot \varphi_{2}(n)$ into Eq. (40) and use (36) for $\varphi_{2, \theta}(n)$. The resulting equation holds if the coefficients of $\varphi_{2}(n+1), \varphi_{2}(n)$, and $\varphi_{2}(n$ -1) are zero. Thus, we obtain

$$
\begin{gathered}
\frac{i a e^{-i \theta}}{2} A(n)=\frac{i a e^{-i \theta}}{2} \omega(n) A(n+1), \\
A_{\theta}(n)-\frac{i a}{2}\left(u_{r}(n)+\frac{i u_{\theta}(n)}{a}\right) A(n)=-\frac{i a}{2}\left(-u_{r}(n)+\frac{i u_{\theta}(n)}{a}\right) A(n), \\
\frac{i a e^{i \theta}}{2} \omega(n-1) A(n)=\frac{i a e^{i \theta}}{2} A(n-1) .
\end{gathered}
$$

From Eqs. (66) and (68), we have that $A(n)=\omega(n) A(n+1)$. Hence, $A(n)=e^{u(n)} F(\theta)$, where $F(\theta)$ is a function of $\theta$ only. Substituting $A(n)=e^{u(n)} b(\theta)$ into Eq. (67), we obtain

$$
b_{\theta}+\left(-\operatorname{iau}_{r}(n)+u_{\theta}(n)\right) b=0 .
$$

Since the function $b$ does not depend on $n$, we have that the coefficient of $b$ in the above equation does not depend on $n$. We obtain the boundary condition (65). Solving Eq. (67) and assuming that the found boundary condition holds, we obtain the expression (64) for $A$.

In Lemma 7, we show that the Lax representations corresponding to the Kelvin transformation (33) and the symmetry (34) are equivalent.

Lemma 7: Let $\varphi_{3}(n)$ be a solution of Eq. (38), then on the boundary $r=a$, a function $\varphi_{4}(n)$ $=A \cdot \varphi_{2}(n)$, where

$$
A=e^{2 i \theta n+u(n)}
$$

is a solution of Eq. (40).

Proof: On the boundary $r=a$, we substitute $\varphi_{4}(n)=A(n, \theta, u, \ldots) \cdot \varphi_{3}(n)$ into Eq. (40) and use (36) for $\varphi_{3, \theta}(n)$. The resulting equation holds if the coefficients of $\varphi_{3}(n+1), \varphi_{3}(n)$, and $\varphi_{3}(n$ $-1)$ are zero. Thus, we obtain

$$
\begin{gathered}
\frac{i a e^{i \theta}}{2} A(n)=\frac{i a e^{-i \theta}}{2} \omega(n) A(n+1), \\
A_{\theta}(n)-\frac{i a}{2}\left(-u_{r}(n)+\frac{4 n}{a}-\frac{i u_{\theta}(n)}{a}\right) A(n)=-\frac{i a}{2}\left(-u_{r}(n)+\frac{i u_{\theta}(n)}{a}\right) A(n), \\
\frac{i a e^{-i \theta}}{2} \omega(n-1) A(n)=\frac{i a e^{i \theta}}{2} A(n-1) .
\end{gathered}
$$

From Eqs. (66) and (68), we have that $A(n)=e^{-2 i \theta} \omega(n) A(n+1)$. Hence, $A(n)=e^{2 i \theta n+u(n)} b(\theta)$, where $b(\theta)$ is a function of $\theta$ only. Substituting $A(n)=e^{2 i \theta n+u(n)} b(\theta)$ into Eq. (67), we obtain

$$
b_{\theta}=0 .
$$

Hence, the function $b$ is a constant. This gives us the expression (64) for $A$.

From the above lemmas, we have the following list of integrable boundary value problems with corresponding Lax pairs. Some of the integrable boundary value problems admit two different Lax pairs. We give both Lax pairs in the list.

The list of integrable boundary value problems for the two-dimensional Toda lattice and corresponding Lax pairs is as follows. 
(1)

$$
\begin{gathered}
r<a, \quad u_{r r}+\frac{1}{r} u_{r}+\frac{1}{r^{2}} u_{\theta \theta}=\omega(n-1)-\omega(n), \\
\varphi_{1, r}(n)=U_{1} \varphi_{1}(n), \quad \varphi_{2, r}(n)=U_{2} \varphi_{2}(n), \\
\varphi_{1, \theta}(n)=V_{1} \varphi_{1}(n), \quad \varphi_{2, \theta}(n)=V_{2} \varphi_{2}(n), \\
r=a, \quad u(n)=2 \operatorname{in} \theta+g(\theta)+k(n), \\
\varphi_{2}=e^{2 i n+g(\theta)} \varphi_{1},
\end{gathered}
$$

where the action of operator $U_{1}$ is given by (30), $V_{1}$ is given by (31), $\tilde{U}_{2}$ is given by (35), and $\tilde{V}_{2}$ is given by (36).

$$
\begin{gathered}
r<a, \quad u_{r r}+\frac{1}{r} u_{r}+\frac{1}{r^{2}} u_{\theta \theta}=\omega(n-1)-\omega(n), \\
\varphi_{1, r}(n)=U_{1} \varphi_{1}(n), \quad \varphi_{3, r}(n)=U_{3} \varphi_{3}(n), \\
\varphi_{1, \theta}(n)=V_{1} \varphi_{1}(n), \quad \varphi_{3, \theta}(n)=V_{3} \varphi_{3}(n), \\
r=a, \quad u_{r}(n)=\frac{2 n}{a}+g(\theta), \\
\varphi_{3}=e^{i a \int g(\theta) \mathrm{d} \theta} \varphi_{1},
\end{gathered}
$$

where the action of operator $U_{1}$ is given by (30), $V_{1}$ is given by (31), $U_{3}$ is given by (37), and $V_{3}$ is given by (38).

$$
\begin{gathered}
r<a, \quad u_{r r}+\frac{1}{r} u_{r}+\frac{1}{r^{2}} u_{\theta \theta}=\omega(n-1)-\omega(n), \\
\varphi_{1, r}(n)=U_{1} \varphi_{1}(n), \quad \varphi_{4, r}(n)=U_{4} \varphi_{4}(n), \\
\varphi_{1, \theta}(n)=V_{1} \varphi_{1}(n), \quad \varphi_{4, \theta}(n)=V_{4} \varphi_{4}(n), \\
r=a, \quad u_{r}(n)=\frac{2 n}{a}+g(\theta), \\
\varphi_{4}=e^{2 i \theta n+u(n)+i a \int g(\theta) \mathrm{d} \theta} \varphi_{1},
\end{gathered}
$$

where the action of operator $U_{1}$ is given by (30), $V_{1}$ is given by (31), $U_{4}$ is given by (39), and $V_{4}$ is given by (40). 
(4)

$$
\begin{gathered}
r<a, \quad u_{r r}+\frac{1}{r} u_{r}+\frac{1}{r^{2}} u_{\theta \theta}=\omega(n-1)-\omega(n), \\
\varphi_{2, r}(n)=U_{2} \varphi_{2}(n), \quad \varphi_{3, r}(n)=U_{3} \varphi_{3}(n), \\
\varphi_{2, \theta}(n)=V_{2} \varphi_{2}(n), \quad \varphi_{3, \theta}(n)=V_{3} \varphi_{3}(n), \\
r=a, \quad u_{r}(n)=-\frac{i}{a} u_{\theta}(n)+g(\theta), \\
\varphi_{3}=e^{-2 i \theta n+i a \int g(\theta) \mathrm{d} \theta} \varphi_{2},
\end{gathered}
$$

where the action of operator $U_{2}$ is given by (35), $V_{2}$ is given by (36), $U_{3}$ is given by (37), and (5) $V_{3}$ is given by (38).

$$
\begin{gathered}
r<a, \quad u_{r r}+\frac{1}{r} u_{r}+\frac{1}{r^{2}} u_{\theta \theta}=\omega(n-1)-\omega(n), \\
\varphi_{2, r}(n)=U_{2} \varphi_{2}(n), \quad \varphi_{4, r}(n)=U_{4} \varphi_{4}(n), \\
\varphi_{2, \theta}(n)=V_{2} \varphi_{2}(n), \quad \varphi_{4, \theta}(n)=V_{4} \varphi_{4}(n), \\
r=a, \quad u_{r}(n)=-\frac{i}{a} u_{\theta}(n)+g(\theta), \\
\varphi_{4}=e^{u(n)+\int g(\theta) \mathrm{d} \theta} \varphi_{2},
\end{gathered}
$$

where the action of operator $U_{2}$ is given by (35), $V_{2}$ is given by (36), $U_{4}$ is given by (39), and $V_{4}$ is given by (40).

Remark 2: The above boundary value problems admit infinitely many solutions. To have a unique solution, one can put additional conditions $u(x, t, 0)=f(x, t)$ and $u(x, t, 1)=g(x, t)$, where $f(x, t)$ is a smooth function compatible with the boundary condition when $n=0$ and $g(x, t)$ is a smooth function compatible with the boundary condition when $n=1$.

\section{REDUCTIONS OF TWO-DIMENSIONAL TODA LATTICE EQUATION}

In this section, we obtain integrable boundary conditions for the sinh-Gordon and Tzitzeica equations as reductions of integrable boundary conditions of the two-dimensional Toda lattice equation.

To reduce the two-dimensional Toda lattice equation to the sinh-Gordon equation, we put periodicity condition $u(n)=u(n+2)$ for all $n$, where $u$ satisfies (29). Then, for $p=u(0)-u(1)$, we have

$$
p_{r r}+\frac{1}{r} p_{r}+\frac{1}{r^{2}} p_{\theta \theta}=4 \sinh p,
$$

the sinh-Gordon equation in the polar coordinates. Only the boundary condition of the problem $u_{r}(n)=-(i / a) u_{\theta}(n)+g(\theta)$ is consistent with the periodicity constraint $u(n+2)=u(n)$. It gives 


$$
p_{r}+\frac{i}{a} p_{\theta}=0
$$

on the boundary $r=a$. Evidently by changing $p=i v$, we get $v_{r r}+(1 / r) v_{r}+\left(1 / r^{2}\right) v_{\theta \theta}=4 \sin v$ and $v_{r}+(i / a) v_{\theta}=0$.

To reduce the two-dimensional Toda lattice equation to the Tzitzeica equation, we put $u(n)$ $=u(n+3)$ and $u(n)=-u(2-n)$. Then, for $q=u(0)$, we have

$$
q_{r r}+\frac{1}{r} q_{r}+\frac{1}{r^{2}} q_{\theta \theta}=e^{2 q}-e^{-q}
$$

the Tzitzeica equation in polar coordinates. Again, only the boundary condition of the problem $u_{r}(n)=-(i / a) u_{\theta}(n)+g(\theta)$ is consistent with periodicity constraints $u(n)=u(n+3)$ and $u(n)=-u(2$ $-n)$. It gives

$$
q_{r}+\frac{i}{a} q_{\theta}=0
$$

on the boundary $r=a$.

\section{SOME SOLUTIONS OF THE BOUNDARY VALUE PROBLEM}

In this section, we give an example of solutions for the special case of the boundary value problem

$$
u_{r r}+\frac{1}{r^{2}} u_{\theta \theta}+\frac{1}{r} u_{r}=\omega(n-1)-\omega(n),\left.\quad u_{r}(n)\right|_{r=a}=\frac{2 n}{a}+g(\theta),
$$

where $\omega(n)=\exp (u(n)-u(n+1))$. We assume that $g(\theta)=0$ and look for a spherically symmetric solution. That is, $u$ is a function of $r$ only. The boundary value problem (79) reduces to

$$
u_{r r}+\frac{1}{r} u_{r}=\omega(n-1)-\omega(n),\left.\quad u_{r}(n)\right|_{r=a}=\frac{2 n}{a} .
$$

Let us introduce new variables $t=\ln (r / a)$ and $v(n, t)=u(n, r)-2 n \ln r$. Then, the boundary value problem (80) becomes

$$
v_{t t}=\bar{\omega}(n-1)-\bar{\omega}(n),\left.\quad v_{t}(n)\right|_{t=0}=0,
$$

where $\bar{\omega}(n)=\exp \{v(n)-v(n+1)\}$. As solutions of the above boundary value problem, we can take even solitons of the Toda lattice equation in one dimension. Following Ref. 19 (see pp. 494-498), the general $N$-soliton solution is given in terms of the data $\left\{c, z_{j}, \gamma_{j}\right\}$ such that

(I) the quantities $z_{j}$ lie in the interval $-1<z_{j}<1$ and are pairwise disjoint;

(II) $\quad e^{-c}=\prod_{j=1}^{N} z_{j}^{2}$;

(III) the quantities $m_{j}(0)=\gamma_{j} / \dot{a}\left(z_{j}\right)$, where $a(z)=\prod_{j=1}^{N} \operatorname{sgn} z_{j}\left(z-z_{j}\right) /\left(z z_{j}-1\right)$ and dot means derivative with respect to $z$, are positive.

The $N$-soliton solution is given by

$$
v(n, t)=c+\ln \frac{\operatorname{det} M(n, t)}{\operatorname{det} M(n-1, t)},
$$

where $M(n, t)$ is a matrix with entries $M_{i j}(n, t)=\delta_{i j}+\sqrt{m_{i}(t) m_{j}(t)}\left(z_{i} z_{j}\right)^{n+1} /\left(1-z_{i} z_{j}\right)$ and $m_{j}(t)$ $=e^{-\left(z_{j}-z_{j}^{-1}\right) t} \gamma_{j} / \dot{a}\left(z_{j}\right), i, j=1, \ldots, N$. 
The even solitons are described by the following lemma.

Lemma 8: Let $N=2 k$ and the data $z_{j}, \gamma_{j}, j=1, \ldots, N$ satisfy $z_{i}=-z_{N-i+1}, \quad \gamma_{i}=-\gamma_{N-i+1}, i$ $=1, \ldots, k$. Then, the $N$-soliton solution (82) is an even function of $t$.

Proof: With our choice of the initial data, the elements of matrix $M(n, t)$, which are symmetric with respect to the "center" of the matrix, are equal. If $t$ is changed to $-t$, then every element of $M(n, t)$ is replaced by the element symmetric to it with respect the center of the matrix. Hence, the determinant of $M(n, t)$ is equal to the determinant of $M(n,-t)$ and $v(n, t)=v(n,-t)$. From $v(n, t)$ $=v(n,-t)$, it follows that $\left.v^{\prime}(t)\right|_{t=0}=0$.

We give an example of the solutions described in the above lemma. For $N=2$, we put $z_{1}$ $=z_{0}, z_{2}=-z_{0}, c=-4 \ln z_{0}$, and $\gamma_{1}=-\gamma_{0}, \gamma_{2}=\gamma_{0}$, where $0<z_{0}<1$ and $\gamma_{0}>0$. Then, the data satisfy conditions (I), (II), and (III) and the conditions of the lemma. With such data, one has the following solution of (81):

$$
v(n, t)=c+\ln \frac{1+\gamma_{0}\left(1+z_{0}^{2}\right) z_{0}^{2 n+1} \cosh \left[\left(z_{0}-z_{0}^{-1}\right) t\right]+\gamma_{0}^{2} z_{0}^{4 n+4}}{1+\gamma_{0}\left(1+z_{0}^{2}\right) z_{0}^{2 n-1} \cosh \left[\left(z_{0}-z_{0}^{-1}\right) t\right]+\gamma_{0}^{2} z_{0}^{4 n}} .
$$

Hence, the boundary value problem (80) has the following solution:

$$
u(n, r)=c+\ln \frac{1+\frac{1}{2} \gamma_{0}\left(1+z_{0}^{2}\right) z_{0}^{2 n+1}\left(r^{\left(z_{0}-z_{0}^{-1}\right)}+r^{-\left(z_{0}-z_{0}^{-1}\right)}\right)+\gamma_{0}^{2} z_{0}^{4 n+4}}{1+\frac{1}{2} \gamma_{0}\left(1+z_{0}^{2}\right) z_{0}^{2 n-1}\left(r^{\left(z_{0}-z_{0}^{-1}\right)}+r^{-\left(z_{0}-z_{0}^{-1}\right)}\right)+\gamma_{0}^{2} z_{0}^{4 n}} .
$$

\section{CONCLUSION}

In the present paper, we apply the method of involutions to boundary value problems for soliton equations on bounded regions. As illustrative models, we consider the Neumann-type boundary value problem on a circle for the Liouville equation and initial boundary value problem for the two-dimensional Toda lattice equation. The Lax representations for the boundary value problems are represented. In the case of Liouville equation in a disk, we failed to find any effective approach to look for a regular solution satisfying the corresponding integrable boundary condition. For the Toda lattice in a cylinder, we have actually a mixed-type problem (time evolution is given by the variable $n$ ). In our opinion, this mixed problem is well posed and can be solved by applying the inverse scattering transformation method. As it was shown in Ref. 18 for the Kadomtsev-Petviashvili (KP) equation on a strip, the Marchenko kernels of two equations connected by an involution are connected by a very simple transformation. In this article, we considered some reductions of the integrable boundary value problems in the case of the twodimensional Toda lattice equation. We also constructed a class of solutions satisfying one of the found boundary conditions.

\section{ACKNOWLEDGMENTS}

The authors thank Scientific and Technological Research Council of Turkey and Turkish Academy of Science for Partial financial support.

${ }^{1}$ E. K. Sklyanin, Funkc. Anal. Priloz. 21, 86 (1987).

${ }^{2}$ B. Gürel, M. Gürses, and I. Habibullin, J. Math. Phys. 36, 6809 (1995).

${ }^{3}$ B. Gürel, M. Gürses, and I. Habibullin, Phys. Lett. A 190, 231 (1994).

${ }^{4}$ V. Adler, B. Gürel, M. Gürses, and I. Habibullin, J. Phys. A 30, 3505 (1997).

${ }^{5}$ I. T. Habibullin and A. N. Vil'danov, in Proceedings of the International Conference "Modern Group Analysis 2000," edited by V. A. Baikov, Ufa, 2001, pp. 80-81.

${ }^{6}$ I. T. Khabibullin and E. V. Gudkova, Funct. Anal. Appl. 38, 138 (2004).

${ }^{7}$ P. N. Bibikov and V. O. Tarasov, Theor. Math. Phys. 79, 570 (1989).

${ }^{8}$ R. F. Bikbaev and V. O. Tarasov, Algebra Anal. 3, 78 (1991).

${ }^{9}$ I. T. Khabibullin, Teor. Mat. Fiz. 86, 43 (1991) [Theor. Math. Phys. 86, 28 (1991)].

${ }^{10}$ V. E. Adler, I. T. Khabibullin, and A. B. Shabat, Teor. Mat. Fiz. 110, 98 (1997) [Theor. Math. Phys. 110, 78 (1997)].

${ }^{11}$ R. F. Bikbaev and A. R. Its, Math. Notes 52, 1005 (1992).

${ }^{12}$ A. B. Borisov and V. V. Kiseliev, Nonlinear World (World Scientific, River Edge, NJ, 1990), Vol. 1, pp. 73-83. 
${ }^{13}$ M. Jaworski and D. Kaup, Inverse Probl. 6, 543 (1990).

${ }^{14}$ A. S. Fokas and D. A. Pinotsis, J. Appl. Math. 17, 323 (2006).

${ }^{15}$ A. S. Fokas, Proc. R. Soc. London, Ser. A 4531411 (1997).

${ }^{16}$ A. B. de Monvel and V. Kotlyarov, Commun. Math. Phys. 253, 51 (2005).

${ }^{17}$ A. Degasperis, S. V. Manakov, and P. M. Santini, Teor. Mat. Fiz. 133, 184 (2002) [Theor. Math. Phys. 133, 1475 (2002)].

${ }^{18}$ E. V. Gudkova and I. T. Khabibullin, Teor. Mat. Fiz. 140, 230 (2004) Theor. Math. Phys. 140, 1086 (2004).

${ }^{19}$ L. D. Fadeev and L. A. Takhtajan, Hamiltonian Methods in the Theory of Solitons (Spinger-Verlag, Berlin, 1987).

${ }^{20}$ A. S. Fokas, J. Math. Phys. 443226 (2003).

${ }^{21}$ A. B. de Monvel, A. S. Fokas, and D. Shepelsky, Commun. Math. Phys. 263, 133 (2006). 